\title{
Ultimate Limit State Design of three-dimensional reinforced concrete structures: a numerical approach
}

\author{
H. Vincent, M. Arquier \\ Strains, Paris, France
}

J. Bleyer, P. de Buhan

Ecole des Ponts ParisTech, Champs-sur-Marne, France

\begin{abstract}
This contribution is addressing the evaluation of the ultimate bearing capacity of massive reinforced concrete structures. It is based on the finite element implementation of both the lower bound static and upper bound kinematic approaches of yield design, adopting the well-known Rankine criterion for modelling the three-dimensional strength properties of plain concrete, while the reinforcing bars are treated by means of an extended homogenization method. Both yield design approaches lead to optimization problems which are solved by resorting to Semi-Definite Programming (SDP) techniques. The whole computational procedure is finally applied to the design of a bridge pier cap, leading to a fairly narrow bracketing of the exact failure load of this kind of structure.
\end{abstract}

\section{INTRODUCTION}

The Ultimate Limit State Design of reinforced concrete structures may be appropriately performed within the theoretical framework of the yield design (Salençon, 2013) or limit analysis (Chen, 1982) theory. As regards the most frequently encountered situation when the structure to be designed is made of an assemblage of 1D (beams or arches) or 2D (plates or shells) structural members, its ultimate bearing capacity may be evaluated from the previous determination of interaction yield criteria involving generalized stresses such as axial-membrane forces and bending moments. This method, which proves particularly attractive from an engineering point of view, has been quite recently used for spatial frame structures (Bleyer \& de Buhan, 2013) and reinforced concrete plates (Bleyer et al., 2015) in combination with efficient convex optimization procedures.

On the other hand, assessing the ultimate load bearing capacity of constructions incorporating massive three-dimensional reinforced concrete components, which can no more be modelled as beams or plates, requires a specific analysis, such as the wellknown "strut-and-tie" model which, in some way, can be related to the static approach of yield design which provides lower bound estimates for the ultimate load bearing capacity of the structure.

With a special attention to evaluating the ultimate shear capacity of reinforced concrete deep beams, both the lower and upper bound methods of yield design have been implemented in the context of a finite element formulation with the help of linear programming techniques (Averbuch \& de Buhan, 1999).
In this study, reinforced concrete was described according to a "mixed modelling" approach, in which plain concrete was modelled as a two-dimensional continuous medium subject to plane stress loading, while the reinforcement bars were treated as one dimensional flexible beams embedded in the surrounding concrete material.

The generalization to the more representative configuration of linear reinforcing inclusions placed into three-dimensional concrete bodies is posing a somewhat serious challenge as regards the possibility of treating such a case in a "1D-3D" mixed modelling approach. Some attempts to circumvent this problem have already been proposed either in the context of the finite element formulation (Llau et al., 2016) or making use of an implicit homogenization method (Nielsen \& Hoang, 2010) or so-called "multiphase model" (Figueiredo et al., 2013).

The present contribution is devoted to applying the previously mentioned multiphase model, initially developed for reinforced soils, to the yield design of three-dimensional reinforced concrete structures. It is based on the combination of the following elements.

$\checkmark$ Formulation of the plain concrete threedimensional strength properties by means of the widely employed Rankine's criterion characterized by the uniaxial tensile and compressive strengths of the concrete.

$\checkmark$ Modelling the strength of each individual reinforcement with its surrounding concrete volume as an anisotropic continuum accounting for the axial strength of the reinforcing inclusion. 
$\checkmark$ Finite element formulation of both the lower bound static and upper bound kinematic approach of yield design based on a discretization of the structure into tetrahedral elements with a piecewise linear variation of the stresses.

$\checkmark$ The final optimization procedure is carried out by means of Semi-definite Programming (SDP).

The whole design procedure will be illustrated on the typical example of evaluating the ultimate bearing capacity of a reinforced concrete bridge pier cap subjected to concentrated vertical loads.

\section{STRENGTH PROPERTIES OF PLAIN AND REINFORCED CONCRETE}

\subsection{Plain concrete strength condition: the Ranki- ne's criterion}

It is generally acknowledged (Chen, 1982; Averbuch \& de Buhan, 1999; Bleyer et al., 2015) that the strength of plain concrete under a three-dimensional multi-axial solicitation is adequately described by a "tension cut-off" Mohr-Coulomb criterion of the form:

$$
F^{c}(\underline{\underline{\sigma}})=\sup \left\{K_{p} \sigma_{M}-\sigma_{m}-f_{c} ; \sigma_{M}-f_{t}\right\} \leq 0
$$

where $\sigma_{M}$ and $\sigma_{m}$ are the major and minor principal components of the stress tensor $\underline{\underline{\sigma}}, f_{t}$ and $f_{c}$ the uniaxial tensile and compressive strengths of the concrete and:

$$
K_{p}=(1+\sin \varphi) /(1-\sin \varphi)
$$

where $\varphi$ is the internal friction angle, which is usually taken equal to $37^{\circ}$.

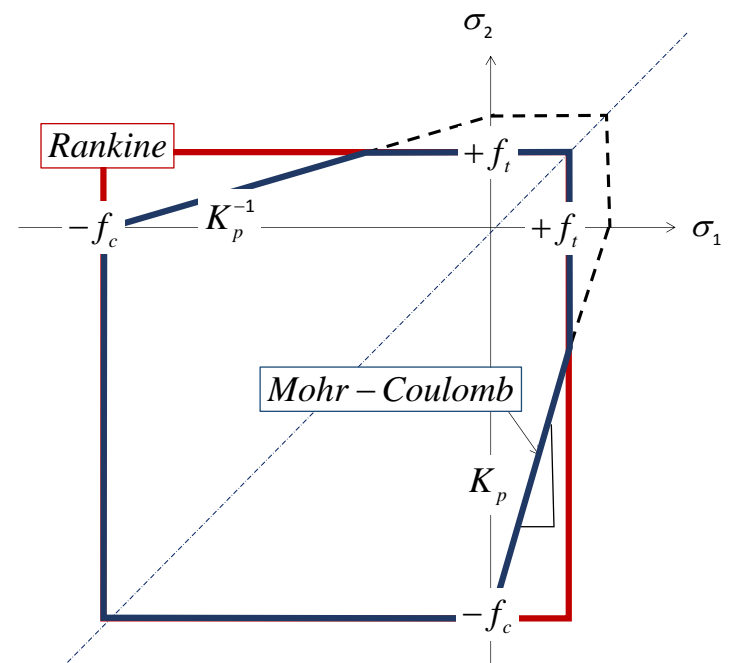

Figure 1. Rankine and tension cut-off Mohr-Coulomb criteria under plane stress conditions

In the following contribution, the simpler Rankine criterion defined by:

$$
F^{c}(\underline{\underline{\sigma}}) \leq 0 \Leftrightarrow-f_{c} \leq \sigma_{m} \leq \sigma_{M} \leq+f_{t}
$$

will be adopted. Figure 1 shows that, under plane stress conditions, the Rankine criterion is slightly conservative with respect to the Mohr-Coulomb one, both criteria being even coincident for $f_{t}=0$. It may also be represented by means of an intrinsic curve in the Mohr-plane, as shown in Figure 2.

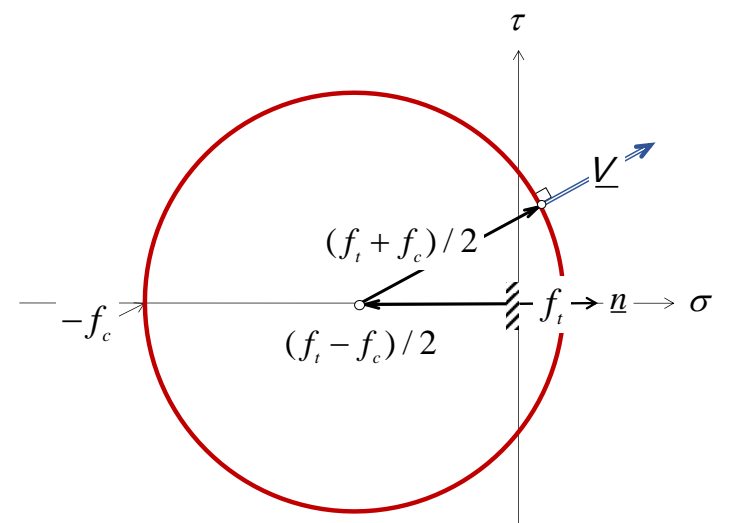

Figure 2. Rankine's intrinsic curve in the Mohr-plane

\subsection{Reinforcements}

The concrete material is reinforced by onedimensional steel bars or rods, the strength condition of which may be simply expressed in terms of axial force $N$ only, thereby neglecting any resistance to shear force $V$ and bending moment $M$ :

$$
-k N_{0} \leq N \leq N_{0}, \quad V=M=0
$$

where $N_{0}$ denotes the tensile resistance of each individual rebar, while $k$ is a non-dimensional parameter ranging from 0 to 1 , expressing the reduced resistance of the reinforcement under compression (buckling for instance).

\subsection{Reinforced concrete as homogenized material}

Some significant zones of the reinforced concrete structure (such as deep beams: see for instance: Averbuch \& de Buhan, 1999) may be reinforced by such uniformly distributed bars (case of stirrups or open frames). Provided that the spacing between two neighboring reinforcements is sufficiently small as compared with the size of the reinforced zone, the latter may be replaced by a zone where the homogenized constituent material obeys a macroscopic strength condition (see de Buhan \& Taliercio, 1991, for composite materials, or Michalowski \& Zhao, 1996 for reinforced soils and Averbuch \& de Buhan, 1999 for reinforced concrete).

This macroscopic strength condition may be expressed as follows:

$$
F^{r c}(\underline{\underline{\sigma}}) \leq 0 \Leftrightarrow\left\{\begin{array}{l}
\underline{\underline{\sigma}}=\underline{\underline{\sigma}}^{c}+\sigma^{r} \underline{e}_{1} \otimes \underline{e}_{1} \\
\text { with } F^{c}\left(\underline{\underline{\sigma}}^{c}\right) \leq 0 \\
\text { and }-k \sigma_{0} \leq \sigma^{r} \leq \sigma_{0}
\end{array}\right.
$$


where $\underline{e}_{1}$ is the unit vector parallel to the reinforcing bar, and $\sigma_{0}$ is defined as the tensile resistance of the bars per unit transverse area:

$$
\sigma_{0}=N_{0} / s^{2}
$$

which may also be expressed as:

$$
\sigma_{0}=A^{s} f_{y}^{s} / s^{2}=\eta f_{y}^{s}
$$

where $f_{y}^{s}$ denotes the uniaxial strength of the bar constituent material (steel) and $A^{s}$ the bar crosssectional area, so that $\eta$ represents the reinforcement volume fraction (see Figure 3 where $s^{2}=A^{s}+A^{c}$ ).

Figure 3 illustrates the macroscopic strength condition (4) expressed on an oriented facet of the homogenized reinforced concrete in the particular case when $k=0$ (no compressive resistance of the reinforcements). Such a geometric representation gives a clear evidence of the strength anisotropy of the homogenized reinforced concrete in exactly the same way as for fiber composite materials.

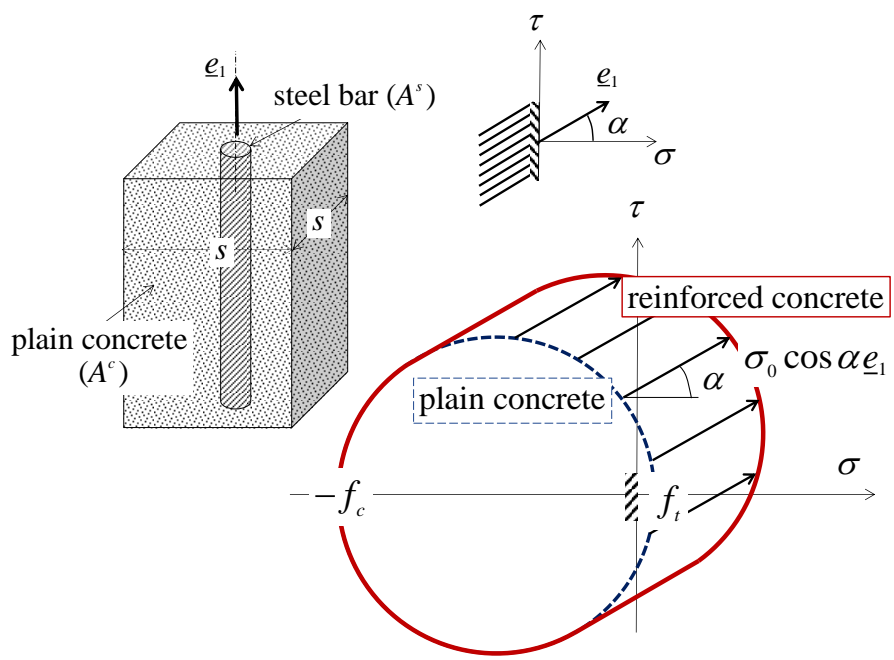

Figure 3. Homogenized strength condition of reinforced concrete relative to an oriented facet

It should be noted that, without any reference to the limit analysis or yield design homogenization theory, some authors (Nielsen \& Hoang, 2010) did make use of a strength criterion quite similar to (5), that is based on an intuitive additive decomposition of the total stress in reinforced concrete zones into stress components relating to the plain concrete and the reinforcements, each one complying with independently specified strength conditions.

\section{MIXED MODELLING OF REINFORCED CON- CRETE}

\subsection{Limitation of the "mixed modelling" approach}

Referring to the frequently encountered situation where only a small number of differently oriented reinforcements are incorporated in the concrete structure (case of longitudinal reinforcements in deep beams for instance), the above mentioned homoge- nization method is no more applicable and the socalled "mixed modelling" approach should be advocated. According to this approach, the reinforcements are treated as 1D structural elements with a strength condition defined by (4) embedded in the concrete material modelled as a 3D continuum, the strength of which is specified by (2).

Unfortunately, this 1D-3D "mixed modelling" approach faces a serious limitation concerning the establishment of equilibrium equations for such a composite system. Indeed, the equilibrium equation at any point of the reinforcing bar may be written as:

$$
\mathrm{d} N\left(x_{1}\right) / \mathrm{d} x_{1}+p\left(x_{1}\right)=0
$$

where $p$ represents the density of axial force exerted by the surrounding concrete material onto the reinforcing bar (Figure 4(a)).

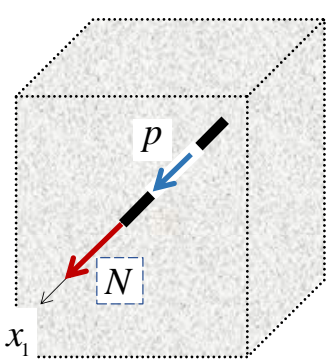

(a)

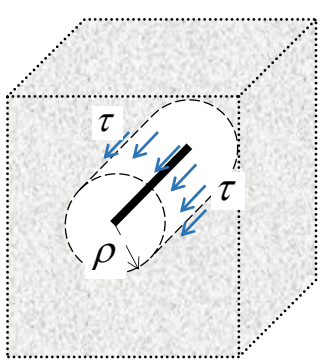

(b)
Figure 4. Interaction forces between concrete and reinforcement in the context of 1D-3D mixed modelling

Now, the impossibility of connecting such a 1D distribution $p$ of interaction forces with the threedimensional stress fields prevailing in the concrete material may be illustrated from the following simple reasoning. Considering a circular cylindrical "control surface" of radius $\rho$ with its axis placed along the reinforcement, as shown in Figure 4(b), the interaction force density $p$ may be obtained from applying along this surface a longitudinal shear stress $\tau$, the average value of which along the circle drawn on this surface at point $x_{1}$, could be expressed as:

$$
\langle\tau\rangle\left(x_{1}\right)=p\left(x_{1}\right) / 2 \pi \rho
$$

According to the latter equation, the shear stress which should be developed in the concrete along the control surface for applying a given value of interaction force density $p$ increases to infinity as the radius $\rho$ tends to zero, so that the stress field in the concrete material would tend to infinity along the reinforcement axis. Such a singularity could possibly be taken into account in the context of a linear elastic behavior of the concrete, but definitely not as soon as yielding and failure of the latter is concerned, since in this case the yield strength condition (3) of the 
concrete would be systematically violated when approaching the 1D reinforcing bar.

\subsection{An extended homogenization model}

Of course, the only fully mechanically consistent and rigorous way of circumventing the above limitation, would be to model each reinforcing bar as a threedimensional volume body. But, on account of the small diameter of such bars along with the sharp contrast between the reinforcing steel and the surrounding concrete in terms of strength properties, this would undoubtedly imply prohibitive computational costs, due for instance to the highly refined discretization required when employing finite element techniques.

An alternative approach for the finite element modelling of 1D steel inclusions in 3D concrete volumes has been recently proposed by Vincent et al. (2017). Considering one individual 1D-inclusion embedded in a 3D-concrete block, a cylindrical volume of concrete with the inclusion placed along its axis is defined, as shown in Figure 5(a). The intuitive idea is to replace the composite cylindrical volume, thus obtained, by a homogenized cylinder, at any point of which the strength condition is defined by Eqs. (4) and (6), where $s$ represents the side of the squared cross-section of the cylindrical volume.

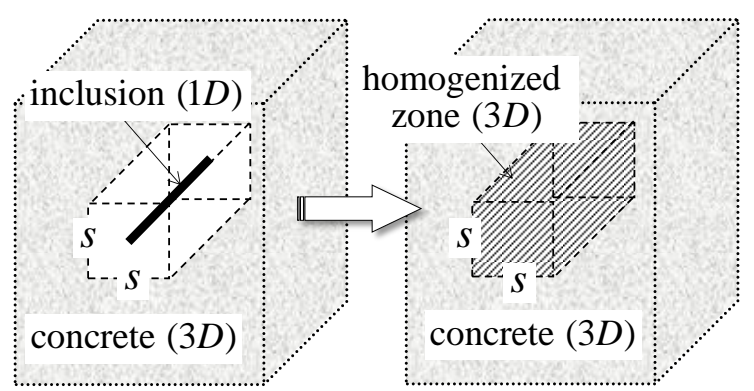

Figure 5. Construction of a homogenized zone around an individual inclusion

The advantage of such a modelling procedure, is that the characteristic size of the homogenized zone (namely $s$ ) is significantly larger than the inclusion's diameter, thus allowing for example a much easier finite element discretization of the reinforced concrete structure as a 3D-3D composite, since a refinement of the mesh around the inclusion is no more required for obtaining accurate and reliable predictions. Of course, the choice of $s$ being arbitrary, it will be necessary to make sure that the results of the computations performed on the basis of this model, remain rather insensitive to the value of $s$, which has been checked in (Vincent et al., 2017).

\section{NUMERICAL LOWER BOUND APPROACH}

\subsection{Statement of the yield design problem}

Assuming that the reinforced concrete structure under consideration is subject to one single loading parameter $Q$, the ultimate or failure load value $Q^{+}$is defined, in the context of the yield design theory, as the maximum value of $Q$ for which one can exhibit any stress field $\underline{\underline{\sigma}}$ :

statically admissible (S.A.) with $Q$, i.e. verifying the equilibrium equation at any point of the structure $\Omega$ :

$$
\operatorname{div} \underline{\underline{\sigma}}(\underline{x})+\rho \underline{F}(\underline{x})=0, \forall \underline{x} \in \Omega
$$

where $\rho \underline{F}$ denotes the body force volume density (material specific weight for example), along with the continuity of the stress-vector across possible stress jump surfaces $\Sigma$ :

$$
[\underline{\underline{\sigma}}(\underline{x})] \cdot \underline{n}(\underline{x})=0, \forall \underline{x} \in \Sigma
$$

as well as the stress boundary conditions associated with the loading $Q$;

and complying with the strength conditions assigned to the plain concrete and reinforced concrete zones of the structure, respectively:

$$
\begin{aligned}
& F^{c}(\underline{\underline{\sigma}}(\underline{x})) \leq 0 \forall \underline{x} \in \Omega^{c} \\
& F^{r c}(\underline{\underline{\sigma}}(\underline{x})) \leq 0 \forall \underline{x} \in \Omega^{r c}, \quad \Omega=\Omega^{c} \cap \Omega^{r c}
\end{aligned}
$$

where $\Omega^{c}$ (respectively $\Omega^{r c}$ ) represents the part of the structure occupied by the plain concrete (resp. by the homogenized reinforced concrete).

\subsection{Finite element formulation and SDP problem}

Applying the lower bound static approach consists in considering S.A. stress fields depending either on a small number of parameters in an analytical approach, or on a large but finite number of stress variables in a numerical approach, such as the finite element method. According to the latter, the geometrical domain $\Omega$ occupied by the threedimensional structure is discretized into $N_{e}$ tetrahedral finite element $\Omega^{e}$, with a linear variation of the stress field inside each element. It is to be noted that there are as many stress tensors attached to any geometrical node of the mesh as there are tetrahedral elements sharing this node as an apex.

It can be shown (Vincent et al., 2017) that the finite element implementation of the lower bound static approach of yield design finally reduces to the following convex optimization problem: 
$Q^{+} \geq Q^{l b}=\operatorname{Max}_{\{\Sigma\}} Q={ }^{T}\{A\}\{\Sigma\}$

subject to $\left\{\begin{array}{l}{[B]\{\Sigma\}=\{C\} \text { equilibrium }} \\ F(\{\Sigma\}) \leq 0 \quad \text { strength criteria }\end{array}\right.$

where $\{\Sigma\}$ is a column-vector which collects all the nodal stress variables associated with the mesh discretization of the structure.

Unlike the equilibrium conditions which involve the total stresses only, the strength criteria in the homogenized reinforced zones concern the partial stresses as shown by (5). While the condition relating to the reinforcement writes in the form of a simple linear constraint, the strength condition of the plain concrete defined by (3) involves the maximal and minimal principal stress components. The latter thus needs a specific treatment so that the optimization problem (12) may be treated as a Semi-definite programming (SDP) optimization problem for which efficient algorithms are available.

\section{UPPER BOUND KINEMATIC APPROACH}

\subsection{Principle of the approach}

The upper bound kinematic approach of yield design is based upon the dualization of the lower bound static one through the virtual work principle (Salençon, 2013). Thus, given any kinematically admissible (K.A.) velocity field $\underline{U}$, the so-called maximum resisting work developed in such a field may be calculated as follows:

$$
\begin{array}{r}
P_{m r}(\underline{U})=\int_{\Omega^{c}} \pi^{c}(\underline{\underline{d}}) \mathrm{d} \Omega^{c}+\int_{\Omega^{r c}} \pi^{r c}(\underline{\underline{d}}) \mathrm{d} \Omega^{r c} \\
\quad+\int_{\Sigma^{c}} \pi^{c}(\underline{n} ; \underline{\underline{V}}) \mathrm{d} \Sigma^{c}+\int_{\Sigma^{r c}} \pi^{r c}(\underline{n} ; \underline{V}) \mathrm{d} \Sigma^{r c}
\end{array}
$$

In the above expression, where $\underline{\underline{d}}$ denotes the strain rate tensor and $\underline{V}$ the velocity jump across the discontinuity surfaces $\Sigma$, the support functions $\pi$, defined as:

$$
\begin{aligned}
& \pi^{c / r c}(\underline{\underline{d}})=\sup \left\{\underline{\underline{\sigma}}: \underline{\underline{d}} ; F^{c / r c}(\underline{\underline{\sigma}}) \leq 0\right\} \\
& \pi^{c / r c}(\underline{n} ; \underline{V})=\sup \left\{(\underline{\underline{\sigma}} \cdot \underline{n}) \cdot \underline{V} ; F^{c / r c}(\underline{\underline{\sigma}}) \leq 0\right\}
\end{aligned}
$$

have the following expressions:

$$
\begin{aligned}
& \pi^{c}(\underline{\underline{d}})=\sum_{k=1}^{3} \sup \left\{f_{t} d_{k} ;-f_{c} d_{k}\right\} \\
& \pi^{c}(\underline{n} ; \underline{V})=\frac{f_{t}+f_{c}}{2}|\underline{V}|+\frac{f_{t}-f_{c}}{2} \underline{V} . \underline{n}
\end{aligned}
$$

for the plain concrete (the second support function relative to a discontinuity may be geometrically calculated from Figure 2), and:

$$
\begin{aligned}
& \pi^{r c}(\underline{\underline{d}})=\pi^{c}(\underline{\underline{d}})+\sup \left\{-k \sigma_{0} d_{11} ; \sigma_{0} d_{11}\right\} \\
& \pi^{r c}(\underline{n} ; \underline{V})=\pi^{c}(\underline{n} ; \underline{V})+\sup \left\{-k \sigma_{0} n_{1} V_{1} ; \sigma_{0} n_{1} V_{1}\right\}
\end{aligned}
$$

for the reinforced concrete. It is worth noting that, unlike for other criteria such as for instance the tension cut-off Mohr-Coulomb condition (1), the support functions (15) and (16) can be calculated for any velocity field and velocity jumps, without it being necessary to impose kinematic restrictions.

Under these conditions, the yield design upper bound kinematic approach states that the ultimate load must satisfy the following inequality, valid for any K.A. velocity field $\underline{U}$ :

$$
Q^{+} \dot{q}(\underline{U}) \leq P_{m r}(\underline{U})
$$

where the left-hand member of the inequality is the virtual work of the ultimate load, thereby producing the following upper bound estimate:

$$
Q^{+} \leq Q^{u b}=P_{m r}(\underline{U}) / \dot{q}(\underline{U}), \text { for } \dot{q}(\underline{U})>0
$$

\subsection{Finite element implementation}

Similarly to what has been previously done for the lower bound static approach (section 4.2.), the reinforced structure $\Omega$ is discretized into $N^{e}$ six-nodded tetrahedral finite element $\Omega^{e}$, with a quadratic variation of the velocity field inside each element and velocity jumps across the triangular facets separating any two adjacent elements.

As shown in (17) the maximum resisting work is greater than the virtual work of the ultimate load. Thus, finding the best upper bound to the ultimate loading of the reinforced structure $\Omega$ can be expressed as the following minimization problem:

$$
Q^{+} \leq Q^{u b}=\operatorname{Min}_{\underline{U}}\left\{P_{m r}(\underline{U}) ; \dot{q}(\underline{U})=1\right\}
$$

Denoting by $\{U\}$ the column-vector collecting all the nodal values of the discretized velocity field, the above minimization problem can be rewritten as:

$$
\begin{aligned}
& Q^{+} \leq Q^{u b}=\operatorname{Min}_{\{U\}}\left\{P_{m r}(\{d\},\{V\})\right\} \\
& \text { subject to }\left\{\begin{array}{l}
\{d\}=[D]\{U\} \\
\{V\}=[E]\{U\} \\
T \\
\{F\}\{U\}=1
\end{array}\right.
\end{aligned}
$$

where $\{d\}$ and $\{V\}$ denoted the column-vectors collecting all the nodal values of the strain rates and velocity jumps associated with the velocity field. This minimization problem is also treated by means of Semi-definite programming (SDP).

\section{PRACTICAL CASE STUDY}

The presented numerical procedure for calculating the ultimate load capacity of 3D structures has been implemented. This section provides the example of a three-dimensional reinforced concrete structure for 
which the ultimate loading capacity is evaluated with a lower bound static approach and an upper bound kinematic approach.

In order to illustrate the efficiency of the implemented 3D yield design procedure, the ultimate bearing capacity of a reinforced concrete bridge pier cap is evaluated.

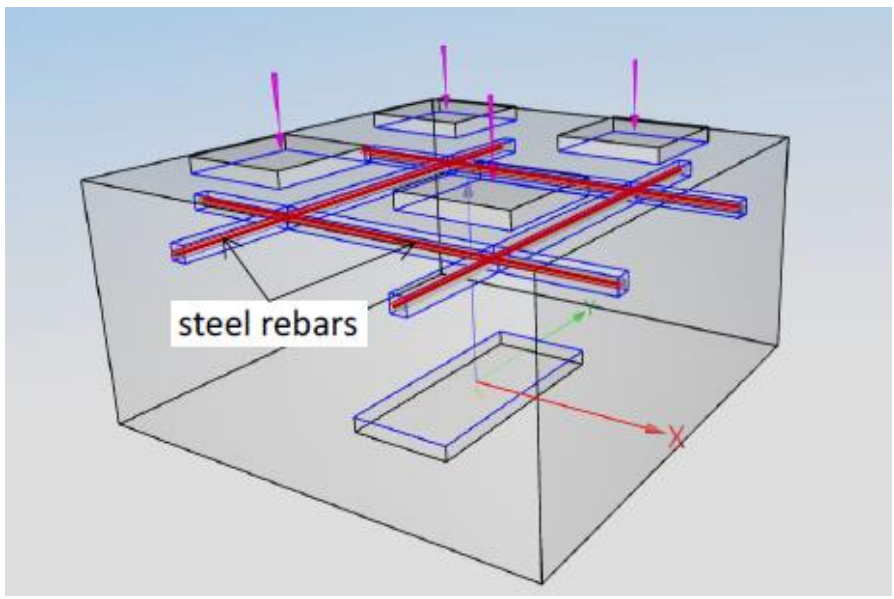

Figure 6. Reinforced concrete pier cap subject to bridge deck loading

The bridge pier cap is modelled as a $3 \times 3 \times 1.5 \mathrm{~m}^{3}$ parallelepipedic concrete block. The finite element lower bound static approach and the upper bound kinematic approach are performed on this structure subject to four vertical loads representing the action of the overlying bridge deck, as shown in Figure 6.

These loadings are applied in the form of a uniform pressure applied on top of small rigid square pads of $0.7 \times 0.7 \mathrm{~m}^{2}$. The interaction with the underlying bridge pier is modelled by imposing a rigid connection on a $1.5 \times 0.7 \mathrm{~m}^{2}$ rectangular area placed at the center of the bottom surface.

The concrete block is made of a homogeneous plain concrete material, with $f_{c}=40 \mathrm{MPa}$ and $f_{t}=0.5 \mathrm{MPa}$. It is strengthened by four steel rebars of diameter equal to $3 \mathrm{~cm}$, placed just below the loading pads as shown in Figure 6, with a uniaxial strength equal to $f_{y}^{s}=400 \mathrm{MPa}$. According to the above described procedure, each of the four rebars is replaced by a homogenized volume of square cross section equal to $s^{2}=0.01 \mathrm{~m}^{2}$.

First, a brief parametric study is carried out to illustrate the convergence of the lower and upper bound approaches towards the exact ultimate load. For this purpose, different meshes with an increasing number of finite elements, have been generated. The results of the analysis are shown in Figure 7 which displays the variations of the lower and upper bound estimates as functions of the mesh refinement characterized by the number of tetrahedral finite elements.

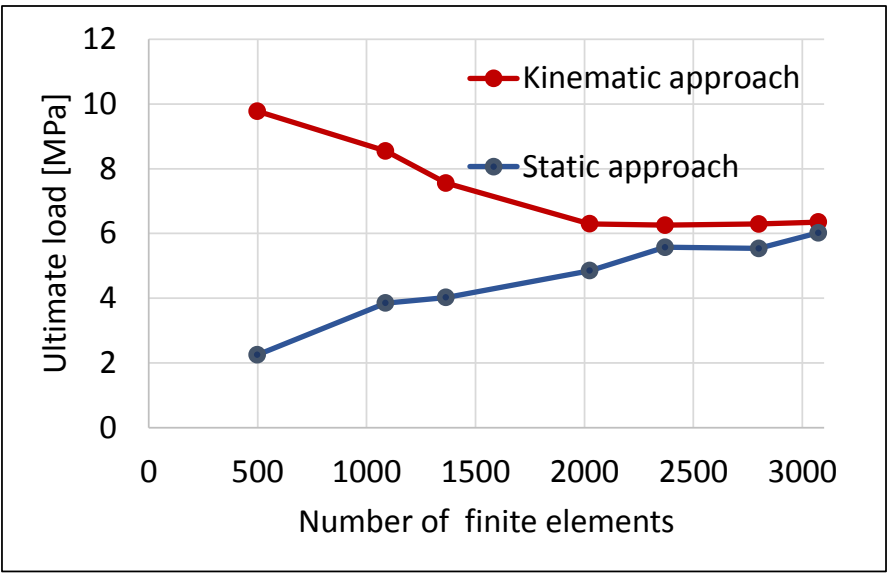

Figure 7. Convergence of the static and kinematic bounds with the increasing number of mesh elements

It may be clearly seen in this figure that the upper and lower bounds are getting closer as the finite element mesh is refined, which that the evaluation of the ultimate load capacity of the structure is becoming more and more accurate. Indeed, the difference between the upper and lower bounds can be interpreted as an error estimator of the result, since the yield design theory ensures that the exact result lies somewhere between the two bounds.

It should be mentioned that the number of tetrahedral finite elements plotted on the horizontal axis corresponds to the kinematic mesh, the static mesh being eight times finer. As the interpolation degree is not the same (linear interpolation for the static approach versus quadratic interpolation for the kinematic approach), the static approach needs to be conducted on finer meshes to converge.

As could be expected, the best results are obtained with the finest meshes, where the structure is discretized in 3073 tetrahedral finite elements for the kinematic approach (24584 for the static approach). However, it is worth noting that the results converge quite rapidly since the relative error is already less than ten percent for kinematic meshes with 2300 elements.

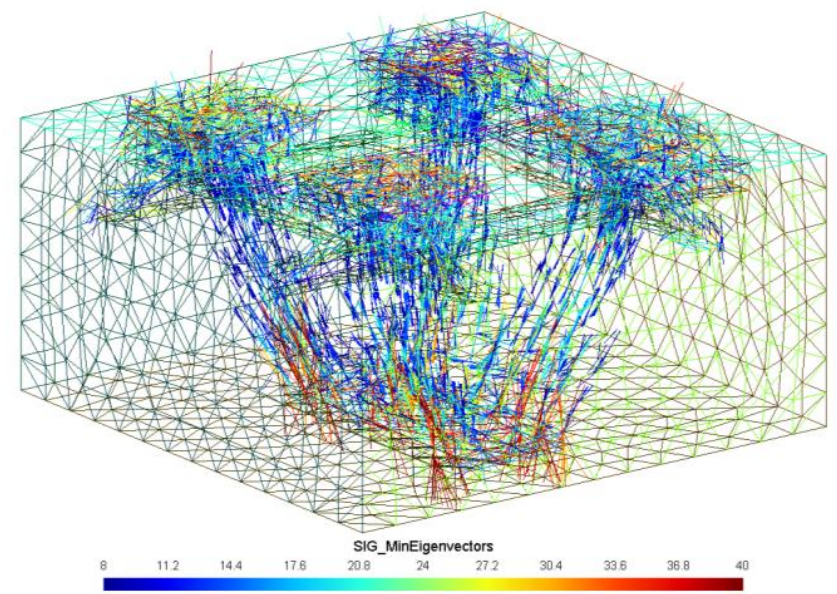

Figure 8. Perspective view of the optimized principal compressive stress field in the concrete 


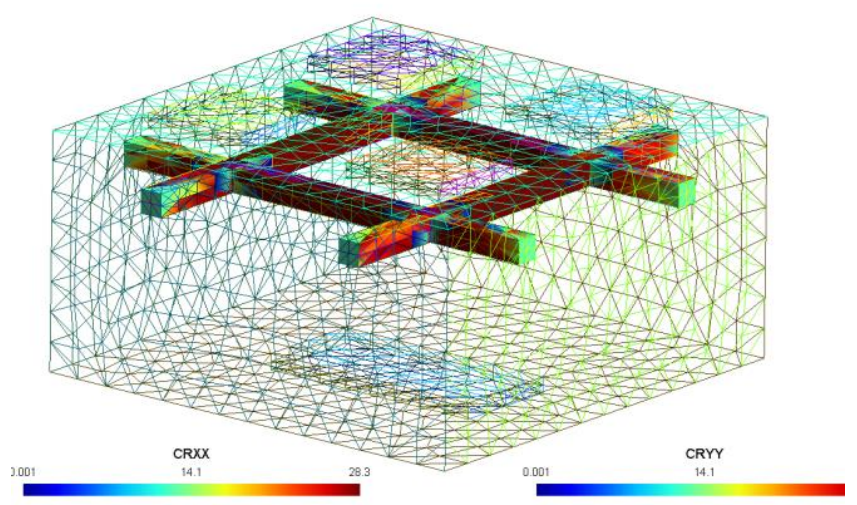

Figure 9. Optimized stress field in the homogenized zone

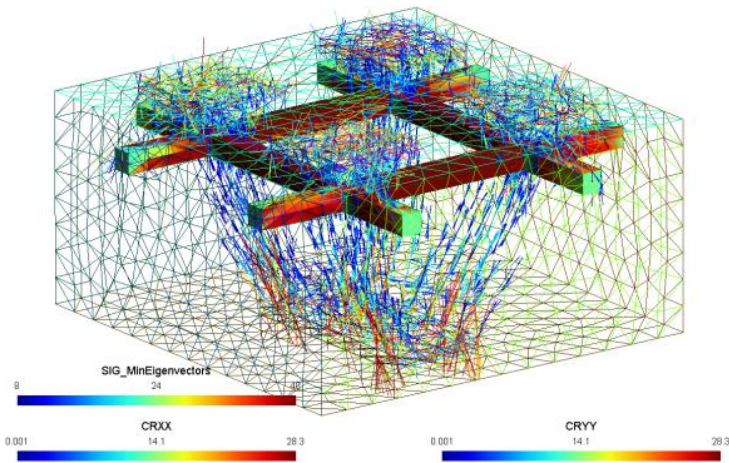

Figure 10. View of the optimized stress field in the whole pier cap

Figure 8 represents the distribution of the principal stresses prevailing in the plain concrete material, while Figure 9 represents the principal tensile stresses in the (homogenized) reinforced zones. Both fields are superimposed in Figure 10.

While the results of the static approach lead to an optimized stress field in the structure which is similar to what the strut and tie method could provide, the kinematic approach gives a clear failure mechanism of the analyzed structure (besides giving an upper bound and thus, an error estimator of the result). The failure mechanism of the reinforced bridge pier cap is shown in Figure 11.

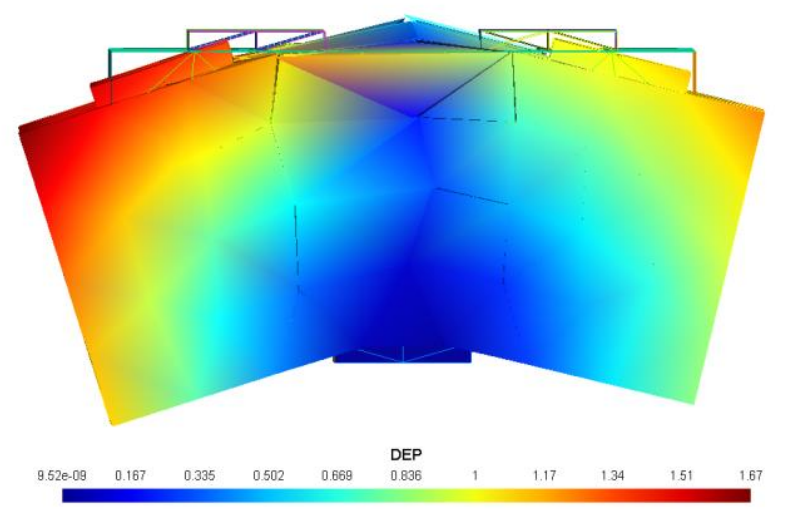

Figure 11. View of the failure mechanism of the pier cap

\section{CONCLUSION}

A specifically dedicated finite element computer code has been set up aimed at producing rigorous lower bound and upper bound estimates for the ultimate load bearing capacity of three-dimensional reinforced concrete structures. It relies upon two recent developments: the numerical formulation of the corresponding optimization problem using Semidefinite Programming, on the one hand, the adoption of a homogenization-based model for describing the mechanical behavior of individual reinforcing inclusions embedded in a surrounding three-dimensional concrete matrix, on the other hand.

The entire procedure may be further extended to other kinds of strength condition for the plain concrete material, such as the already mentioned tension cut-off Mohr-Coulomb criterion (1), which may prove more realistic for capturing the strength properties of concrete, notably under isotropic confining stresses. This will imply to take kinematic restrictions into account in the calculations of the support functions $\pi$ and thus of the maximum resisting work

\section{REFERENCES}

Averbuch D., de Buhan P., 1999. Shear Design of Reinforced Concrete Deep Beams: A Numerical Approach, Jl. of Structural Engineering, 125(3): 309-318.

Bleyer J., de Buhan P., 2013. Yield surface approximation for lower and upper bound yield design of 3D composite frame structures, Computers and Structures, 129: 86-98.

Bleyer J., Pham D.T., de Buhan P., 2015. Failure design of high-rise concrete panels under fire loading, Engineering and Computational Mechanics, 168, EM4: 178-185.

de Buhan P., Taliercio A., 1991. A homogenization approach to the yield strength of composite materials, European Journal of Mechanics, A/Solids, 10(2): 129-150.

Chen W.F., 1982. Plasticity in reinforced concrete, McGrawHill, New-York.

Figueiredo M.P., Maghous S., Filho A.C., 2013. Threedimensional finite element analysis of reinforced concrete structural elements regarded as elastoplastic multiphase media, Materials and Structures, 46: 383-404.

Llau A., Jason L., Dufour F., Baroth J., 2016. Finite element modelling of 1D steel components in reinforced and prestressed concrete structures, Engineering Structures, 127: 769-783.

Michalowski P.V., Zhao A., 1996. Failure of fibre-reinforced granular soils, Jl. Geotech. Eng., ASCE, 122(3): 226-234.

Nielsen M.P., Hoang L.C., 2010. Limit Analysis and Concrete Plasticity, CRC Press, Taylor \& Francis.

Salençon J., 2013. Yield Design, ISTE Ltd, Wiley, London.

Vincent H., Arquier M., Bleyer J., de Buhan P., 2017. Yield design numerical analysis of three-dimensional reinforced concrete structures. $4^{\text {th }}$ Int. Conf. on Mech. Models in Struct. Eng., 29 nov-01 dec., Madrid, Spain. 